\title{
Face allotransplantation for various types of facial disfigurements: A series of five cases
}

\author{
Ömer Özkan MD ${ }^{1}$ (1) | Özlenen Özkan MD ${ }^{1}$ | Mehmetcan Ubur ${ }^{1}$ | \\ Necmiye Hadimioğlu ${ }^{2}$ | Melike Cengiz ${ }^{2}$ | ismail Afşar ${ }^{3}$
}

${ }^{1}$ Department of Plastic and Reconstructive Surgery, Akdeniz University Faculty of Medicine, Antalya, Turkey

${ }^{2}$ Department of Anaesthesiology and Reanimation, Akdeniz University Faculty of Medicine, Antalya, Turkey

${ }^{3}$ Dentist, Anaplastologist, Private practice, Antalya, Turkey

\section{Correspondence}

Ömer Özkan, Plastik ve Rekonstrüktif Cerrahi Anabilim Dalı, Akdeniz Üniversitesi Hastanesi, B Blok kat 2, Antalya 07059, Turkey.

Email: omozkan@hotmail.com
Background: The aim of this report is to present our long-term experiences with a series of 5 face-transplanted patients in terms of surgical aspects and postoperative outcomes, and to describe possible salvage strategies in case of difficulties.

Methods: Five patients, 4 receiving full-face transplantation and 1 undergoing partial transplantation at our institution were included. The patients were aged between 19 and 54 years. Two had extensive burn scars to the face, and 3 had suffered gunshot injuries. The post-transplant induction immunosuppressive regimen included ATG combined with tacrolimus, mycophenolate mofetil, and prednisone, while maintenance was provided by the last 3. We focused on patient summaries including their etiologies, preoperative preparations, surgical techniques, immunosuppressive regimen, postoperative courses, revisional surgeries, together with challenges including acute rejection episodes, and immunosuppressive drug complications.

Results: No re-surgery due to vascular compromise was required in any case. One of the 5 patients was eventually lost due to complicated infectious and metabolic events at the end of post-transplantation month 11 . The other 4 patients were still alive, with a mean follow-up time of 53 months and had satisfactory functional transplants and cosmetic appearance.

Conclusions: Face transplantation still involves challenges and many issues including compliance and psychological maturity of patients, the risk of opportunistic infections and malignancies still need to be resolved for it to be accepted as a safe procedure. Surgical rescue procedures considering ideal timing should be kept in mind strictly as one of the most important issues in case of unexpected events.

\section{1 | INTRODUCTION}

Face allotransplantation is a recent restorative procedure aiming to replace function and esthetic appearance for patients with severe disfigurement which cannot be managed by conventional reconstructive procedures. The feasibility of the procedure has been confirmed through $>30$ cases in various countries. However, the procedure was initially regarded as a fantastical and even utopian concept in almost all countries in which was performed (Devauchelle et al., 2006; Guo et al., 2008; Lantieri et al., 2008; Siemionow et al., 2008). However, as the numbers of patients have increased, more difficulties and challenges have also been encountered. More refinements and better results have now been achieved in all new cases, especially in centers with higher levels of experience (Giatsidis, Sinha, \& Pomahac, 2017; Khalifian et al.,
2014; Lantieri et al., 2016; Petruzzo et al., 2011, 2012; Pomahac et al., 2012; Siemionow, Gharb, \& Rampazzo, 2013).

In addition to difficulties involving surgical techniques, the most serious handicap is the use of immunosuppressive drugs, which are well known to increase the risk of opportunistic infections, metabolic complications and malignancies. As in all surgical procedures, there must be exit strategies in vascularized composite tissue allotransplantations in case of unfavorable developments (Ozkan et al., 2017). In the earliest period, experience with solid organ transplantations was employed in order to overcome problems related to the use of immunosuppressive drugs, since there was considerably more experience with these. However, increasing experience with face transplantation has led to an expansion of the relevant literature, and several undesirable and catastrophic cases have been reported, together with many 
highly satisfactory ones. Unfortunately, losing a patient is a particularly catastrophic outcome in these procedures because they are not lifesaving.

Nevertheless, face transplantation has become a clinical reality with improvements in immunosuppressive regimens together with surgical technical refinements. Since 2012, we have performed 5 face transplantations, 4 total and 1 partial. In this report, we present our long-term experiences with this series in terms of surgical aspects and postoperative outcomes, and describe possible salvage strategies in case of difficulties.

\section{2 | PATIENTS AND METHODS}

Based on the vascularized composite tissue transplantation directive (No. 13984) issued in 2011 by the General Directorate of Curative Services of the Turkish Ministry of Health (http://www.saglik.gov.tr/ TR,11273/kompozit-doku-nakli-merkezleri-yonergesi.html), the internal scientific committee of Akdeniz University, Turkey, investigated and verified patients' suitability for possible transplantation and selected patients recorded on the national transplantation list after evaluation by the National Scientific Committee on Vascularized Composite Tissue Transplantation. In 2011, 6 candidates for face transplantation (5 male and 1 female) were approved by the committee in the light of the criteria laid out in the directive. We also drew up a detailed informed consent form and a legal contract. This contract referred to all potential complications associated with this potentially life-threatening and nonlife-saving procedure, especially well-known or foreseeable drugrelated complications, such as severe infections, diabetes, kidney failure, lymphoma and bone necrosis.

Five patients, all male, aged between 19 and 54 years (mean 31.2), underwent surgery between January 2012 and December 2013. Descriptive details of the patients were summarized in Table 1. Four full-face and 1 partial face transplantation were performed. The etiology was burn deformity in 2 patients (Figure 1; patient 1 and 2), and gunshot injury in the remaining 3 patients (Figure 1; patient 3, 4, and 5).

All patients had previously undergone several conventional reconstructive procedures. The national organ procurement organization identified suitable brain-dead donors based on specified criteria. Our surgeons were divided into 2 teams, one for the recipient and the other for donor procurement, each with specific objectives during the procedures.

\section{1 | Surgical procedures}

All transplanted patients presented with no panel reactive antibodies. No patients were evaluated for donor specific antibodies. Cytomegalovirus (CMV) Ig $\mathrm{M}$ was negative in all patients, and CMV Ig $\mathrm{G}$ was positive. Similarly, Epstein-Barr virus (EBV) IG M was negative, and EBV Ig $\mathrm{G}$ was positive. Both complement-dependent cytotoxic crossmatch (CDC) and flow cytometric crossmatch (FCXM) were negative. Donor and recipients were blood compatible with 5 to total HLA-A, -B, -DR mismatches. In each instance, 1 team performed facial allograft harvesting. The harvested allografts were perfused with University of
Wisconsin solution and transported in an ice-water slurry. Prosthetists on this team prepared a mask before allograft recovery, and this was applied after allograft removal in order to maintain donor body integrity. In synchronization with the donor operation, another team removed the deformed skin and facial tissue remnants and isolated all necessary motor and sensorial nerve branches and neck vessels from the recipient. Full-face transplantation were performed, including all facial skin and facial musculature, except for the eyelids and the nasal bone in patient 1 (Figure 2, above left, above right), all facial skin and facial musculature, together with the eyelids (Figure 2, below left), the nasal bone, the anterior half of the hairy scalp and the right auricle in patient 2, all facial skin and facial musculature, the upper and lower jaws and the nasal bone in patient 3 and all facial skin and facial musculature, both eyelids, the nasal bone, the maxilla, part of the mandible, the base of the tongue and the anterior part of the scalp in patient 4. Partial face transplantation was performed, including the entire nose and upper lip in patient 5.

All facial allografts exhibited complete perfusion after implantation, and neural repair was provided as required. Arterial anastomoses were performed between external carotid artery matches in an end-to-end manner in all 4 full-face transplantations. Venous anastomoses were performed between the external jugular veins in an end-to-end manner and internal jugular vein matches in an end-to-side manner, bilaterally. In the partial face transplantation case, arterial anastomoses were performed between external carotid artery matches in an end-to-side manner on 1 side and facial artery matches on the other side. Venous anastomoses were performed between the external jugular vein matches on 1 side and between the internal jugular vein matches in an end-to-side manner on the other side. Facial nerve coaptations (Figure 2 , below right) and sensorial neural repair were performed in all cases, with the exception of the last partial transplant case, in which no neural coaptation was performed. In the first 2 and the partial (last) transplant patients, only the nasal bones were fixed for skeletal reconstruction, while in the third and fourth patients both Le-Fort III segment fixations and central mandibular segment fixations were carried out. The patients were initially treated in the intensive care unit for several days, and were subsequently transferred to the surgical ward until discharge (Figure 1). Except for 1 case, donor tissues were provided by various centers at considerable distances from our own institution. Cold ischemia time ranged between 1 and $3 \mathrm{~h}$, and total ischemia time between 2 and $6 \mathrm{~h}$.

\section{2 | Immunosuppressive protocol}

Antithymocyte globulin and prednisolone were administered in the initial induction period. Antithymocyte globulin was initiated intraoperatively at a dose of $2.5 \mathrm{mg} / \mathrm{kg}$ per day. Prednisolone was started on the day of surgery, at $1000 \mathrm{mg}$, and tapered down to $20 \mathrm{mg} 1$ week postoperatively. Tacrolimus was started (Prograf, $0.2 \mathrm{mg} / \mathrm{kg}$ per day with blood levels between 15 and $20 \mathrm{ng} / \mathrm{ml}$ ) on day 4, and antithymocyte globulin was stopped on days 7-10, depending on achievement of the desired tacrolimus blood levels. Maintenance treatment consisted of prednisolone $(20 \mathrm{mg} /$ day $)$ tapering to $10 \mathrm{mg} /$ day 6 months 


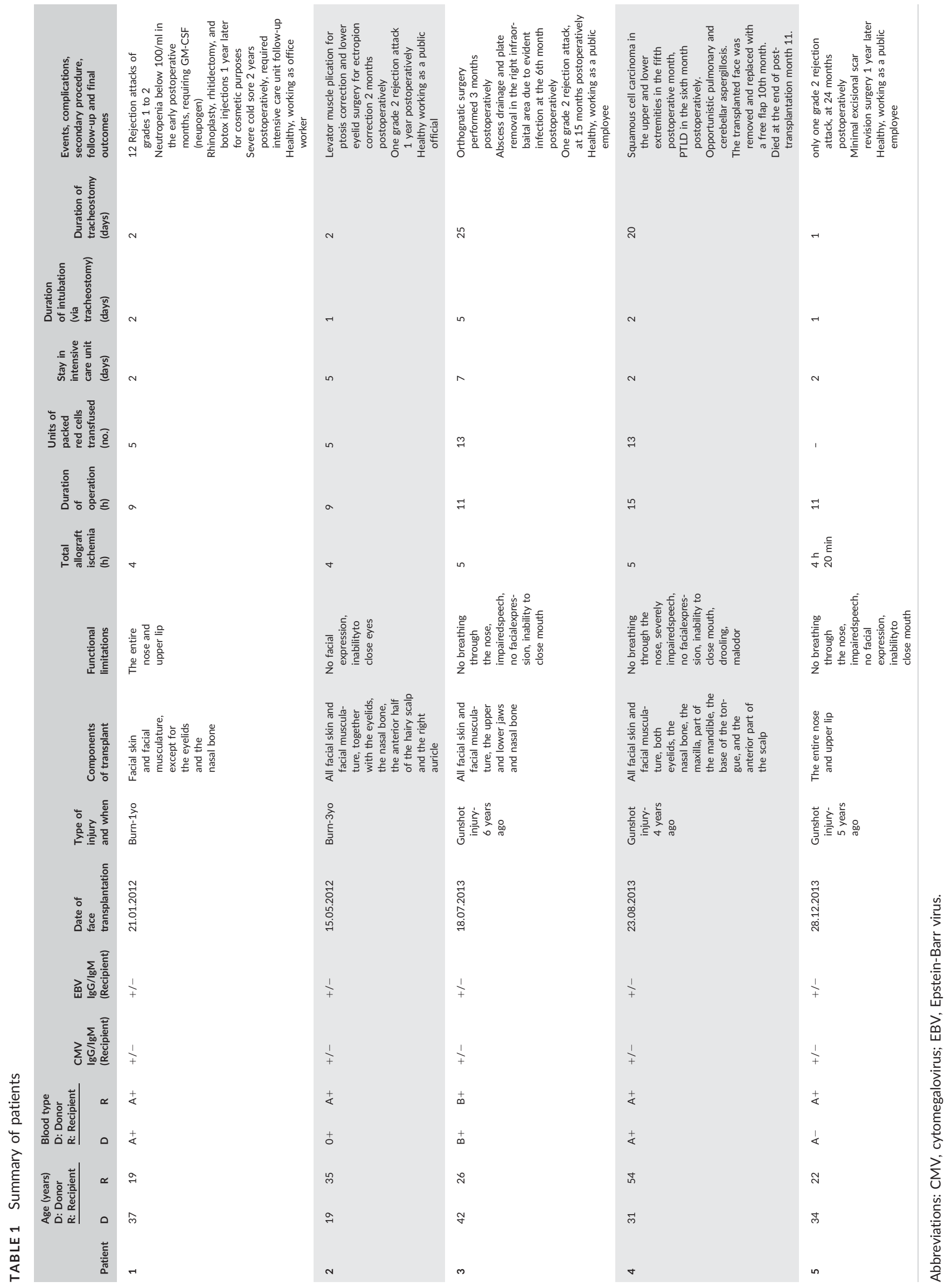



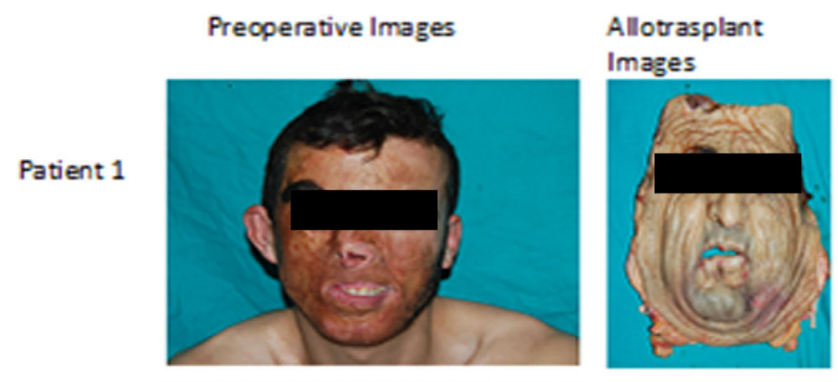

Postoperative Images
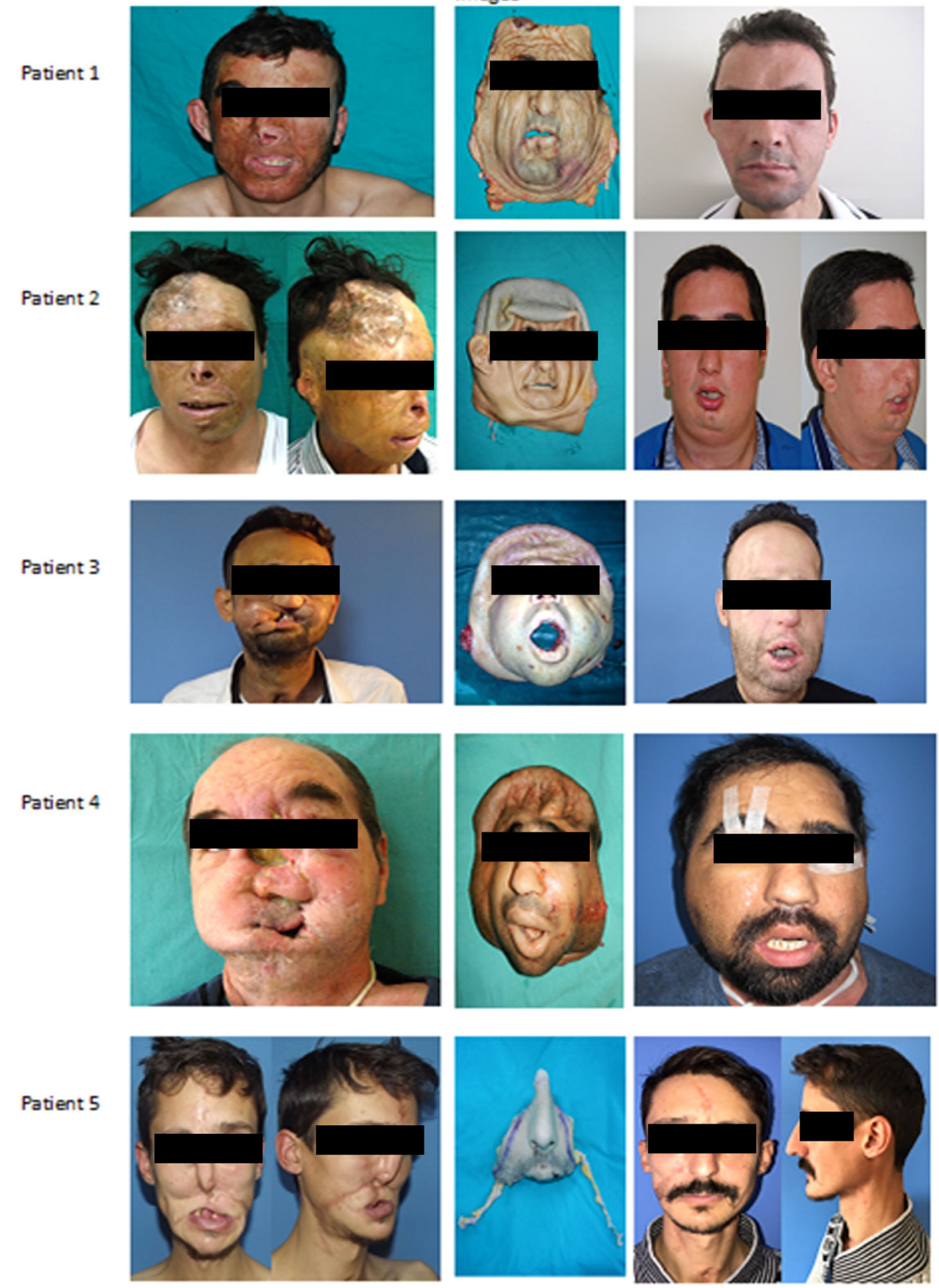

FIGURE 1 Photographs of the patients before transplantation and transplanted part and several months after surgery. Left column: preoperative view. Patient 1 had whole face severe burn scar especially in the midface; patient 2 had whole face burn scar including bilateral eyelids together with right auricle and half of the anterior hairy scalp; patient 3 had severe facial deformity especially in the midface due to gun-shot injury; patient 4 had severe whole face deformity due to gun-shot injury; patient 5 had midface deformity. Middle column: intraoperative harvested face transplant. Whole face harvested for patient 1, patient 2 (included bilateral eyelids, right auricle, and anterior hairy scalp), 3 (included upper and lower jaws), patient 4 (included bilateral eyelids, upper and lower jaws, anterior hairy scalp, and tongue base) and partial face harvested for patient 5 (included total nose and upper lip). Right column: postoperative appearance at 11 months (patient 1), 24 months (patient 2), 15 months (patient 3), 3 months (patient 4), and 35 months (patient 5)

postoperatively, tacrolimus (blood levels between 15 and $20 \mathrm{ng} / \mathrm{ml}$ for the first 3 months, tapered to $7-10 \mathrm{ng} / \mathrm{ml} 6$ months postoperatively) and mycophenolate mofetil (MMF) (2 g/day). Patient 4 required a modified protocol due to development of severe complications.

\section{3 | Anti-infection and anti-coagulation prophylaxis}

Wide spectrum antibiotic therapy was administered for the first 10 days postoperatively to prevent infectious complications. Sulfadoxine pyrimethamine was given to prevent Pneumocystiscarinii pneumonia. 

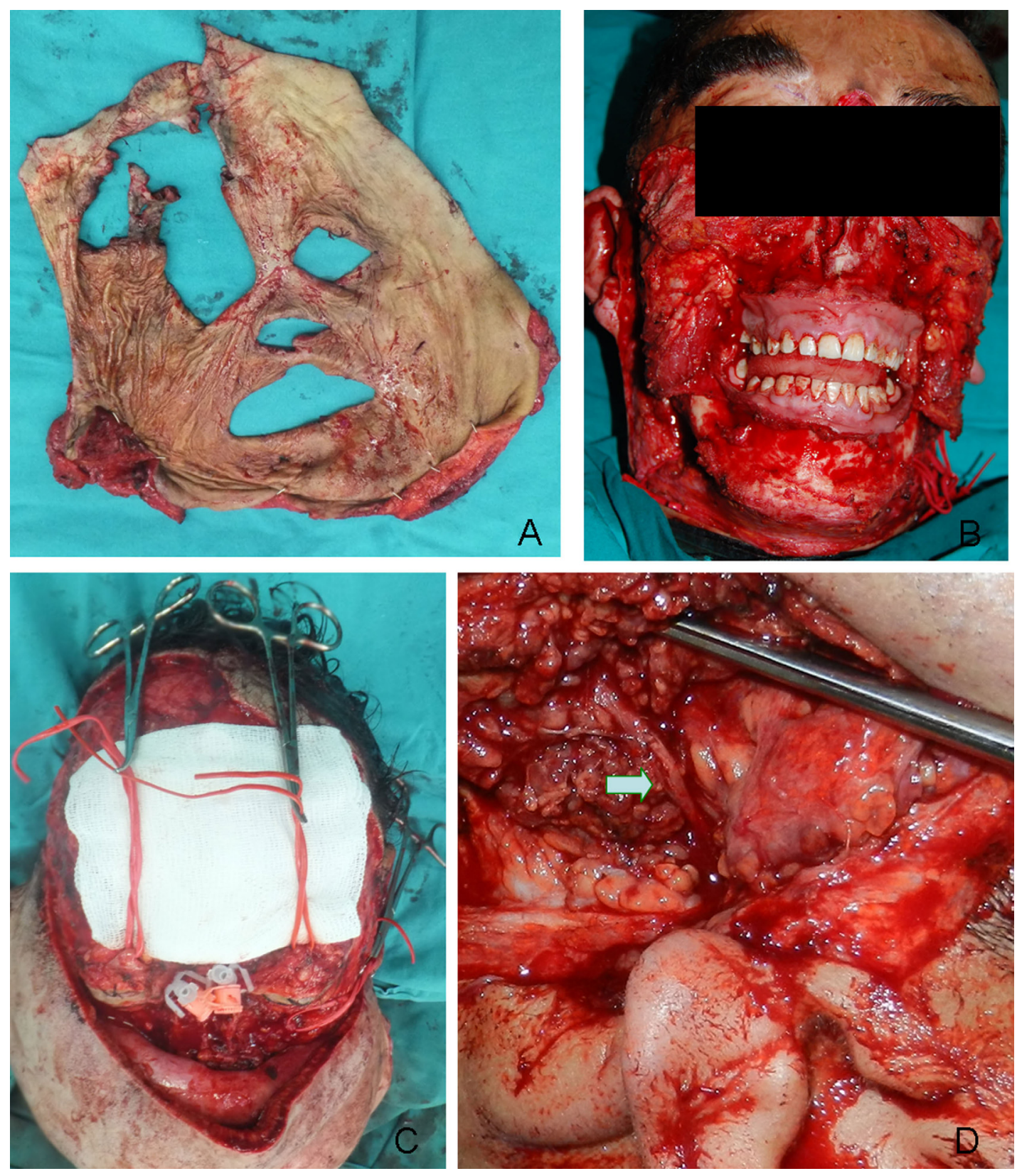

FIGURE 2 Intraoperative photographs for surgical technique. Above left: excised deformed tissue from the recipient in patient 1. Above right: view of the recipient after excision of some part of the deformed tissue (patient 1). Below left: adaptation of the transplanted tissue to the recipient face (during eyelid levator muscle reattachment in the second patient). Below right: facial nerve coaptation in patient 4 (arrow shows repaired facial nerve)

Oral nystatin drops were administered for fungal prophylaxis, and oral valacyclovir tablets for CMV prophylaxis. Antithrombotic prophylaxis was administered with subcutaneous heparin for 5 days postoperatively.

Mucosa biopsies were scheduled every 2 weeks for 3 months in the transplanted oral mucosa, and then monthly for 6 months. Skin biopsies close to incision scars were collected from the most inconspicuous area every month for the first 6 months, and then every 3 months. Biopsy samples were graded according to the Banff 2007 scale. Tracheotomies were performed postoperatively in all cases, and were removed within 1 week to 1 month. All patients had access to frequent counseling from psychiatrists and social workers. Although patients' satisfaction levels were generally high, they had not been evaluated with any scoring system at the time of writing of this report. However, we planned to do this in the near future, and the results will be the subject of a separate report. No re-surgery due to vascular compromise was required in any case.

\section{3 | RESULTS}

Patients were followed-up for between 9 and 65 months (mean 44.8 months). The long-term outcomes and events are summarized in 
Patient 1 (Burn injury)

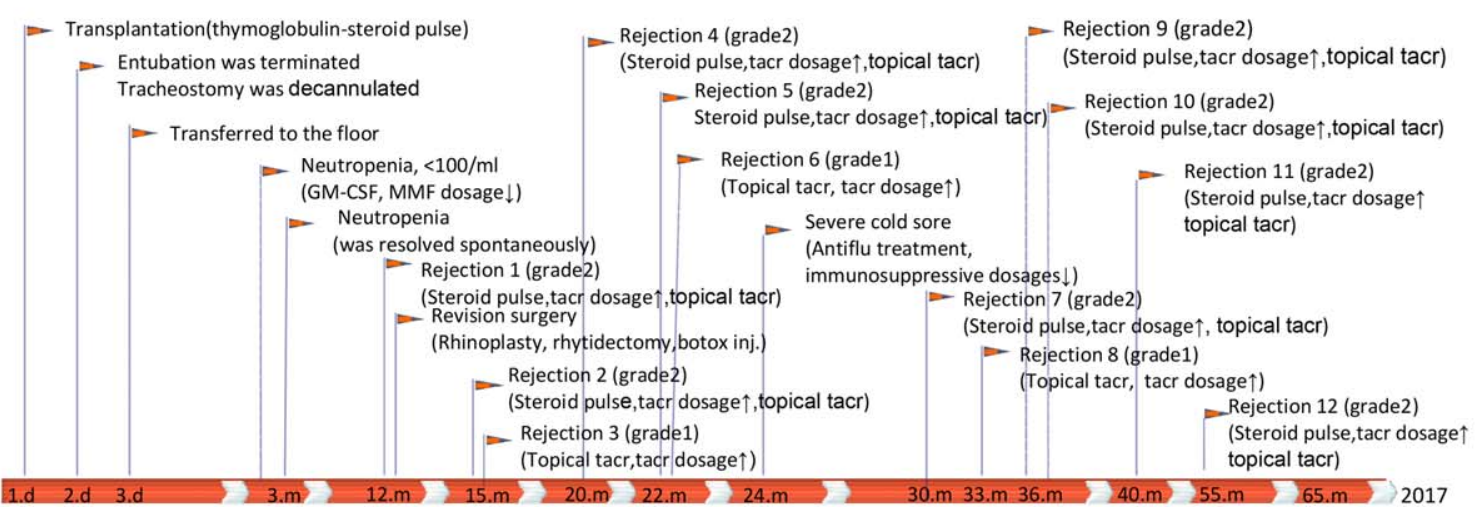

Patient 2 (Burn injury)

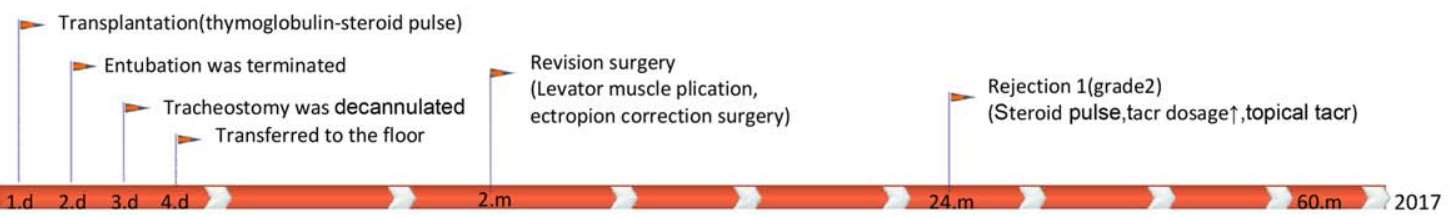

Patient 3 (Gunshot injury)

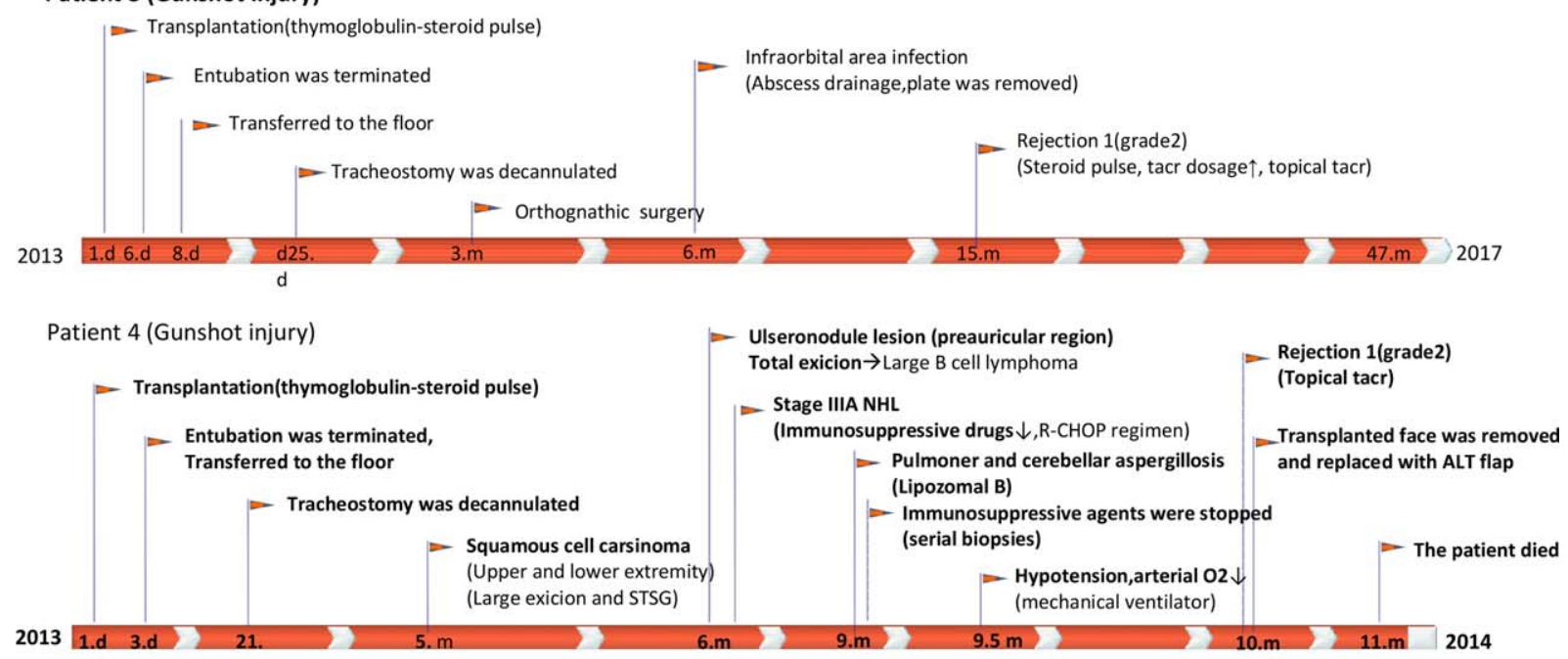

Patient 5 (Gunshot injury)

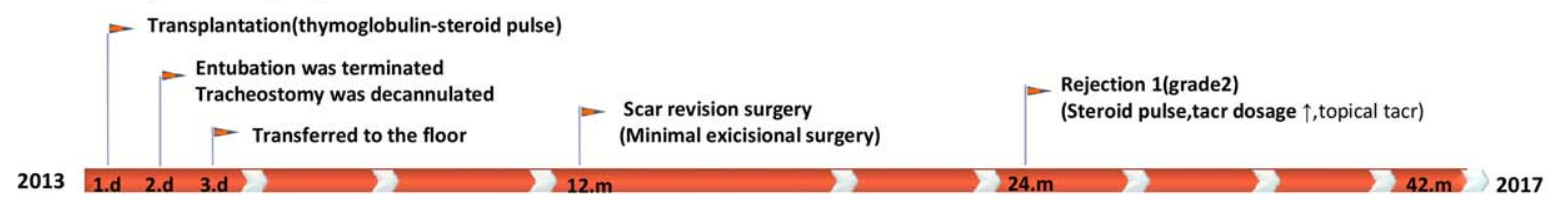

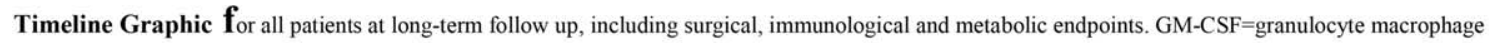
colony stimulating factor. MMF=mycophenolate mofetil. Tacr-tacrolimus. STSG=split- thickness skin graft, ALT: Anterolateral Thigh Flap, d=day(s). $\mathrm{m}=$ month(s)

FIGURE 3 Timelines for the patients at long-term follow-up

Table 1 and Figure 3. Of 5 transplanted patients, 1 had since died (Figure 1; patient 4). In patient 4 , although the early postoperative period was uneventful, squamous cell carcinoma developed in the upper and lower extremities in the fifth postoperative month, and post-transplant lymphoproliferative disorder (PTLD) occurred in the sixth month. Malignancies were treated, involving both surgical and medical approaches. The patient developed opportunistic pulmonary and cerebellar aspergillosis. In order to reduce the adverse effects, and the metabolic and immunological load, the transplanted face was removed and replaced with a free flap. Although the early 
postoperative period was promising, with the transferred flap surviving totally and all vital signs and general status appearing to be improving, the patient was eventually lost due to complicated infectious and metabolic events at the end of post-transplantation month 11 .

In patient 1 neutropenia below 100/ml requiring GM-CSF (neupogen) occurred in the early postoperative months. The MMF dosage was adjusted based on the severity of neutropenia, ranging between none and $500 \mathrm{mg}$ twice a day. Bone marrow biopsy revealed nonspecific findings, and the problems resolved spontaneously 3 months postoperatively.

All patients had at least 1 episode of acute cell-mediated rejection higher than grade 2 between 6 months and 5 years postoperatively. All were easily detected by observation of erythema and edema and were confirmed by pathological examination. Patient 1 had no rejection attack until 12 months postoperatively, but then suffered 12 rejection attacks of grades 1 (detected incidentally at routine pathological examination) to 2 , very possibly due to noncompliance with immunosuppressive drugs. The patient had been generally compliant in the last year and has experienced only one grade 2 rejection attack. All grade 2 attacks resolved with administration of steroid pulse together with a temporary increase of tacrolimus dosage. Grade 1 attacks resolved with topical use of tacrolimus and also an increase in tacrolimus drug dosage. The incidence of rejections decreased with close follow-up, adjustment of drug dosages and close psychological support. Patients 2, 3, and 5 suffered only one grade 2 rejection attack each, 1 year, 15 months, and 24 months, postoperatively, respectively. All resolved with topical application of tacrolimus cream, a temporary increase in tacrolimus dosage and, in patients 3 and 5, additional administration of pulse steroid therapy. Patient 4 experienced rejection attacks after reduction of immunosuppressive drugs due to the severe complications described above.

Only the first patient suffered a severe infectious complication (infectious complication developed in patient 4 due to use of chemotherapeutic agents).The patient suffered a severe cold sore requiring anti-flu treatment, and was hospitalized, developed severe viral pneumonia and required intensive care unit follow-up 2 years postoperatively. No agent was isolated from samples. Immunosuppressive drug dosages were reduced, together with administration of empiric antibacterial, antifungal and antiviral prophylaxis.

Although long-term results were not available for patient 4, satisfactory facial nerve movements and sensorial innervations were observed in all the other full face-transplanted patients. Sensorial innervations began as early as 2 months and facial movements from the third months on, with further improvement over time. Sensorial recovery was observed at 4 months postoperatively, although there was no nerve repair in the partial face-transplanted patient.

Some revisional surgeries were required. In patient 1 , rhinoplasty, rhitidectomy, and botox injections were performed 1 year subsequently for cosmetic purposes. Patient 2 underwent levator muscle plication for ptosis correction and lower eyelid surgery for ectropion correction 2 months postoperatively. Patient 3 underwent orthognatic surgery 3 months postoperatively to provide better occlusion. At 6 months postoperatively, abscess drainage and plate removal were required in the right infraorbital area due to significant infection. Minimal excisional scar revision surgery was performed after 1 year in the case of patient 5 .

All surviving patients adapted normally to social life. Our first patient was employed in office work in our university 6 months after surgery. Patient 2 married 3 years after surgery and is currently working as a public official. Patient 3 is currently working as a public employee in the patient's previous post. That patient married 2 years postoperatively and has a healthy child from that marriage. Patient 5 is currently working as a public employee.

\section{4 | DISCUSSION}

Similarly to hand transplantations (Özkan et al., 2014), the aim of facial transplantation is to enhance recipients' quality of life. Although the public demand for face transplantation is increasing, the procedure is still far more complex and difficult than other forms of organ transplantation, due to a lack of sufficient experience in all aspects of the subject. There has also been a debate concerning the medical ethics of face transplantation (Coffman \& Siemionow, 2014).

Most transplanted faces in the literature have experienced at least 1 episode of acute rejection, as in all of our cases (Fischer et al., 2016). One of the most challenging problems is to establish a balance between preventing acute rejection and simultaneously avoiding potentially life-threatening complications. The currently generally used conventional regimens and doses are quite similar in most well-known centers. We have used a similar protocol in all our vascularized composite tissue transplantation cases and in all solid organ transplantations.

Although it was once thought that facial vessels alone could not perfuse the entire face and anterior scalp and that superficial temporal vessels must be included in full-face allografts (Meningaud, Paraskevas, Ingallina, Bouhana, \& Lantieri, 2008; Wang et al., 2007), in our all 5 cases including full face and midface defects, bilateral facial arteries (extended to the external carotid arteries) nourished the entire transplant and no other vascular structure was needed for blood supply as also indicated by Pomahac et al. (2012) No vascular anastomosis revision was required in any of our cases. From a functional point of view, a remarkably good recovery of sensibility has been documented in all patients (Uysal et al., 2016). Recovery of motor function has enabled patients to perform facial mimics in all full face transplantations. In the partial midface case, only upper lip musculature was included in the transplant, and although no neural repair was provided, functional lip movement has been achieved, most probably due to muscular neurotization.

Malignancy is 1 potential catastrophic complication observed in transplanted patients (Diaz-Siso, Sosin, Plana, Rodriguez, 2016; Kanitakis et al., 2015; Piselli et al., 2014; Singavi, Harrington, \& Fenske, 2015). Two separate lesions appeared a few months after transplantation in our fourth case. These were diagnosed as squamous cell carcinoma. These cancers are closely associated with level of immunosuppression, and several studies have reported that withdrawal of or 
even a decrease in immunosuppression can slow progression or reverse the skin tumor. Since the interval between the occurrence of these lesions and diagnosis of lymphoma was quite short, we were unable to evaluate the late course of the squamous cell carcinomas.

Post-transplant lymphoproliferative disorders are the second most common malignancies in transplanted patients (Diaz-Siso, Sosin, Plana, Rodriguez, 2016; Kanitakis et al., 2015; Piselli et al., 2014; Singavi, Harrington, \& Fenske, 2015). These are less responsive to conventional treatment, and outcomes are generally poor. Although reducing or withdrawing immunosuppression is the first step in treatment and is of great potential benefit in prognosis in solid organ transplants, we lack sufficient experience with vascularized composite tissue transplanted patients.

The etiology of the opportunistic infections that are most observed with immunosuppressive drug-related complications in allotransplantations may again differ from that in solid organ allotransplantations (Knoll et al., 2013; Weissenbacher, Hautz, Pratschke, \& Schneeberger, 2013). Interaction between the external environment and transplanted vascularized composite tissue facilitates the emergence of various pathogens. Facial allografts which include the paranasal sinuses possess specific potential focuses for opportunistic infections. Exposure to direct mechanical and surgical trauma, including postoperative manipulative procedures such as irrigative cleansing, gastrointestinal tube insertions and dental rehabilitation, are other possible predisposing factors.

The high risk of life-threatening fungal infection is still a significant and major challenge. Aspergillus is the second most common fungal infection, after Candida, and one that can involve nearly all organs (Barchiesi et al., 2015; Hoyo et al., 2014; Meng et al., 2014). Due to nonspecific clinical manifestations, early detection is almost impossible. It is usually diagnosed when it is already well disseminated, and associated mortality rates can be as high as $90-100 \%$. If life is to be saved, early detection is crucial for the administration of effective antifungal treatment and, if possible, removal of infected original lesions. The risk is especially high in patients receiving steroid therapy, particularly in high doses during rejection attacks. True microbiological diagnosis may not always be possible, and treatment may proceed based on clinical findings, radiological imaging, and indirect blood test results, such as positive antigen levels.

Surgical rescue procedures in vascularized composite tissue transplantations are an important issue in case of unexpected events (Ozkan et al., 2017). In extremity transplantations, the final possible salvage procedure is amputation of the allotransplanted part, which will not result in a worse situation than that pertaining prior to the transplantation procedure. However, in face transplantation, the situation is exceedingly challenging. Most patients will have already exhausted all conventional reconstructive options before face transplantation is added to the list. Salvage procedure may be required in several situations, including surgical complications (failures due to technical or early immunological and ischemic causes), psychological non-compliance on the part of the patient, the need to withdraw immunosuppressive drugs due to opportunistic infections, heavy metabolic complications and malignancies, and chronic rejection. In failures due to technical reasons, requiring the patient who has already received urgent face transplantation to undergo another transplantation procedure seems to be the only acceptable solution. However, the patient should be reconstructed using conventional methods, at least in the early period. In all other situations, facial re-transplantation should not be regarded as an option. All possible efforts should be made to reconstruct patients using conventional methods. Although in the case of partial face transplantation the reconstructive procedure may be acceptable compared with the preoperative appearance, even when total face transplantation is performed using all reconstructive tools the outcome may still not be comparable with the preoperative appearance. The challenge involves not only the choice of reconstructive method, but also the medical status of the patient in a life-threatening condition. As in our case, it may not be possible to achieve an ideal stable situation, and the procedure may have to be performed under highly risky conditions. The benefit to risk ratio should be discussed in detail, and the responsibility must be shared between all the relevant specialties.

Although we used similar immunosuppressive drugs in all our cases, the most logical hypothesis concerning our fourth patient, who died due to sequential postoperative complications, may be a predisposition and high sensitivity to immunosuppressive complications that could not be envisaged preoperatively.

Patient compliance is of the utmost importance in face transplantation, as in all other allotransplantations (Chang \& Pomahac, 2013; Coffman, 2015; Coffman, Gordon, \& Siemionow, 2010). We observed rare acute rejection attacks in all of our vascularized composite transplanted patients consistent with the literature knowledge, except for our first face transplantation case, which was noncompliant with the use of immunosuppressive drugs. That patient suffered 12 rejection attacks, which all resolved well with minor interventions including topical use, temporary increase of drug doses and pulse steroids. This subject has been generally compliant in the last year, with the help of psychological support, and has experienced only one grade rejection attack.

Another important problem with face transplantation is the possibility of metabolic and hormonal problems again associated with the use of immunosuppressive drugs, such as diabetes, increased creatinine levels, Cushing's syndrome, avascular necrosis, and so on. Although we encountered such no complications, any physician wishing to perform any kind of allotransplantation should nevertheless remember the possibility of all these complications occurring.

In almost all facial transplantation cases, revisional procedures will be required (Aycart et al., 2015). Although minor scar revisions are mostly associated with cosmetic appearance, together with any esthetic procedures such as rhinoplasty or blepharoplasty or any kind of injection such as filler, fat, or botox, the main consideration and challenge concerns the functional aspect. Revisional surgery in cases including the eyelid or orthognatic correction in transplants including the jaw are the most commonly applied functional revisional interventions in full face transplantations. They are particularly important to patient satisfaction with the procedure.

An approach involving at least 2 teams, both well experienced in microsurgery, one for the donor and another for the recipient, is essential. Assistant team members are also of very great importance, in terms 
of preoperative organization, equipment organization for far distance donor harvesting, intraoperative assistance, postoperative tissue transportation and, of course, postoperative monitoring of the transplanted tissue and patient care. Intensive care unit experience is necessary for the early postoperative period. Surgical details such as neurovascular dissections, maxillofacial bony integration and 3 dimensional adaptation require little more additional experience for an experienced head and neck microsurgeon. Long pedicular dissection of the transplanted tissue and a precise bony segment with good preoperative planning are important to ensure comfortable neurovascular repair and easy bony reattachment. Any team that is keen to perform face transplantation should remember that psychological rehabilitation together with social integration of the patient are as important as the success of the procedure in order to achieve the desired and satisfactory results.

\section{5 | CONCLUSION}

Face transplantation still poses challenges, and many issues still need to be resolved for it to be accepted as a safe procedure. In addition to the surgical complexity, lifelong use of immunosuppressive drugs with a distinct risk of complications is the major challenge involved with the procedure. Compliance and psychological maturity of candidate patients are highly important issue that should be meticulously evaluated preoperatively. Nevertheless, with more detailed patient evaluation and appropriate selection, face transplantation should maintain its position at the top of the reconstructive ladder in the field of reconstructive surgery with its unique principle of replacing like with like to achieve highly satisfactory results.

\section{ACKNOWLEDGMENT}

This study was supported by the Akdeniz University Scientific Research Projects Unit. We thank them for their support.

\section{CONFLICT OF INTEREST}

We declare that we have no conflict of interest.

\section{REFERENCES}

Aycart, M. A., Alhefzi, M., Kueckelhaus, M., Fischer, S., Bueno, E. M. Pomahac, B. (2015). Secondary revisions after facial transplantation: Optimizing functional and aesthetic outcomes. Plastic and Reconstructive Surgery, 136, 152-153.

Barchiesi, F., Mazzocato, S., Mazzanti, S., Gesuita, R., Skrami, E., Fiorentini, A., Singh, N. (2015). Invasive aspergillosis in liver transplant recipients: Epidemiology, clinical characteristics, treatment, and outcomes in 116 cases. Liver Transplantation, 21, 204-212.

Chang, G., Pomahac, B. (2013). Psychosocial changes 6 months after face transplantation. Psychosomatics, 54, 367-371.

Coffman, K. L. (2015). Psychiatric evaluation of the face transplant candidate. Current Opinion in Organ Transplantation, 20, 222-228.

Coffman, K. L., Gordon, C., Siemionow, M. (2010). Psychological outcomes with face transplantation: Overview and case report. Current Opinion in Organ Transplantation, 15, 236-240.
Coffman, K. L., Siemionow, M. Z. (2014). Ethics of facial transplantation revisited. Current Opinion in Organ Transplantation, 19, 181-187.

Devauchelle, B., Badet, L., Lengelé, B., Morelon, E., Testelin, S., Michallet, M., ... Dubernard, JM. (2006) First human face allograft: Early report. Lancet, 368, 203-209.

Diaz-Siso, J. R., Sosin, M., Plana, N. M., Rodriguez, E. D. (2016). Face transplantation: Complications, implications, and an update for the oncologic surgeon. Journal of Surgical Oncology, 113, 971-975.

Fischer, S., Lian, C. G., Kueckelhaus, M., Strom, T. B., Edelman, E. R., Clark, R. A., ... Pomahac, B. (2014). Acute rejection in vascularized composite allotransplantation. Current Opinion in Organ Transplantation, 19, 531-544.

Giatsidis, G., Sinha, I., Pomahac, B. (2017). Reflections on a decade of face transplantation. Annals of Surgery, 265, 841-846.

Guo, S., Han, Y., Zhang, X., Lu, B., Yi, C., Zhang, H., ... Li, H. (2008). Human facial allotransplantation: A 2-year follow-up study. Lancet, 372, 631-638.

Hoyo, I., Sanclemente, G., de la Bellacasa, J. P., Cofan, F., Ricart, M. J., Cardona, M., ... Cervare, C. (2014). Epidemiology, clinical characteristics, and outcome of invasive aspergillosis in renal transplant patients. Transplant Infectious Disease, 16, 951-957.

Kanitakis, J., Petruzzo, P., Gazarian, A., Testelin, S., Devauchelle, B., Badet, L., ... Morelon, E. (2015). Premalignant and malignant skin lesions in two recipients of vascularized composite tissue allografts (face, hands). Case Reports in Transplantation, 2015, 356459.

Khalifian, S., Brazio, P. S., Mohan, R., Shaffer, C., Brandacher, G., Barth, R. N., Rodriguez, E. D. (2014). Facial transplantation: The first 9 years. Lancet, 384, 2153-2163.

Knoll, B. M., Hammond, S. P., Koo, S., Issa, N. C., Tullius, S. G., Baden, L. R., ... Marty, F. M. (2013). Infections following facial composite tissue allotransplantation-single center experience and review of the literature. American Journal of Transplantation, 13, 770-779.

Lantieri, L., Grimbert, P., Ortonne, N., Suberbielle, C., Bories, D., Gil-Vernet, S., ... Hivelin, M. (2016). Face transplant: Long-term follow-up and results of a prospective open study. Lancet, 388, 13981407.

Lantieri, L., Meningaud, J. P., Grimbert, P., Bellivier, F., Lefaucheur, J. P., Ortonne, N., ... Wolkenstein, P. (2008). Repair of the lower and middle parts of the face by composite tissue allotransplantation in a patient with massive plexiform neurofibroma: A 1-year follow-up study. Lancet, 372, 639-645.

Meng, X. C., Jiang, T., Yi, S. H., Xie, P. Y., Guo, Y. F., Quan, L., ... Shan, H. (2014). Renal aspergillosis after liver transplantation: Clinical and imaging manifestations in two cases. World Journal of Gastroenterology, 20, 18495-18502.

Meningaud, J. P., Paraskevas, A., Ingallina, F., Bouhana, E., Lantieri, L. (2008). Face transplant graft procurement: A preclinical and clinical study. Plastic and Reconstructive Surgery, 122, 1383-1389.

Özkan, Ö., Demirkan, F., Özkan, Ö., Dinçkan, A., Hadimioglu, N., Tuzuner, S., ... Gunseren F. (2011). The first (double) hand transplantation in Turkey. Transplantation Proceedings, 43, 3557-3560.

Ozkan, O., Ozkan, O., Doğan, U., Yılmaz, V. T., Uysal, H., Undar, L., ... Ramazanoglu, A. (2017). Consideration of difficulties and exit strategies in a case of face allotransplantation resulting in failure. Microsurgery, 37, 661-668.

Petruzzo, P., Kanitakis, J., Badet, L., Pialat, J. B., Boutroy, S., Charpulat, R., ... Morelon, E. (2011). Long-term follow-up in composite tissue allotransplantation: In-depth study of five (hand and face) recipients. American Journal of Transplantation, 11, 808-816. 
Petruzzo, P., Testelin, S., Kanitakis, J., Badet, L., Lengelé B., Girbon, J. P., ... Dubernard, J. M. (2012). First human face transplantation: 5 years outcomes. Transplantation, 93, 236-240.

Piselli, P., Verdirosi, D., Cimaglia, C., Busnach, G., Fratino, L., Ettorre, G. M., ... Serraino, D. (2014). Epidemiology of de novo malignancies after solid-organ transplantation: Immunosuppression, infection and other risk factors. Best Practice \& Research Clinical Obstetrics \& Gynaecology, 28, 1251-1265.

Pomahac, B., Pribaz, J., Eriksson, E., Bueno, E. M., Diaz-Siso, J. R., Rybicki, F. J., ... Tullius, S. G. (2012). Three patients with full facial transplantation. The New England Journal of Medicine, 366, 715-722.

Siemionow, M., Gharb, B. B., Rampazzo, A. (2013). Successes and lessons learned after more than a decade of upper extremity and face transplantation. Current Opinion in Organ Transplantation, 18, 633-639.

Siemionow, M., Papay F., Alam D., Bernard S., Djohan R., Gordon C., ... Fung J. (2009). Near-total human face transplantation for a severely disfigured patient in the USA. Lancet, 374, 203-209.

Singavi, A. K., Harrington, A. M., Fenske, T. S. (2015). Post-transplant lymphoproliferative disorders. Cancer Treatment and Research, 165, 305-327.
Uysal, H., Özkan, Ö., Barçın, E., Şenol, U., Tombak, K., Özkan, Ö. (2016). Referred facial sensation on the hand after full face transplantation. Neurology, 86, 836-839.

Wang, H. Y., Li, Q. F., Zheng, S. W., Chen, B., Li, Y. P., Tang, L. J., Chang, T. S. (2007). Cadaveric comparison of two facial flap-harvesting techniques for alloplastic facial transplantation. Journal of Plastic, Reconstructive \& Aesthetic Surgery, 60, 1175-1181.

Weissenbacher, A., Hautz, T., Pratschke, J., Schneeberger, S. (2013). Vascularized composite allografts and solid organ transplants: Similarities and differences. Current Opinion in Organ Transplantation, 18, 640-644.

How to cite this article: Özkan Ö, Özkan Ö, Ubur M, Hadimioğlu N, Cengiz M, Afşar I. Face allotransplantation for various types of facial disfigurements: A series of five cases. Microsurgery. 2018;38: 834-843. https://doi.org/10.1002/micr.30272 